\title{
Sarcoma de Kaposi em pacientes portadores de HIV
}

\author{
Kaposi's sarcoma in HIV patients
}

Sarcoma de Kaposi en pacientes con VIH

Paulo Sérgio da Paz Silva Filho ORCID: https://orcid.org/0000-0003-4104-6550 Centro Universitário Maurício de Nassau, Brasil E-mail: pauloosergio1@outlook.com

Alaíde Silva Lemos

ORCID: https://orcid.org/0000-0003-4121-6724

Centro Universitário Uninovafapi, Brasil E-mail: laid_mel13@hotmail.com

Camila Pinheiro Santiago Silva ORCID: https://orcid.org/0000-0001-7650-246X Universidade Ceuma, Brasil

E-mail: camilapss91@gmail.com

Maria Clara Gomes Silva

ORCID: https://orcid.org/0000-0002-8469-1034

Centro Universitário UniFacid Wyden, Brasil

E-mail: mc.claragomes.mc@hotmail.com

Perla Maria Antão de Alencar Carvalho ORCID: https://orcid.org/0000-0001-8372-1265

Universidade de Fortaleza, Brasil

E-mail: perlinhantao@hotmail.com

Alexandra Rodrigues Cardoso

ORCID: https://orcid.org/0000-0002-7277-4668

Centro Universitário Maurício de Nassau, Brasil E-mail: alexandrarodcar@gmail.com Juniel Marques de Oliveira

ORCID: https://orcid.org/0000-0002-0557-6872 Universidade Estadual do Piauí, Brasil E-mail: junielmrqs.88@gmail.com

Fernanda Maria Silva

ORCID: https://orcid.org/0000-0002-0504-9896 Universidade Regional do Cariri, Brasil E-mail: fernandamsmv@gmail.com

Ana Alinne Gomes Da Penha

ORCID: https://orcid.org/0000-0001-9253-1199 Universidade Regional do Cariri, Brasil E-mail: anaalinne.nurse@gmail.com

Samyra Lima Ferreira

ORCID: https://orcid.org/0000-0002-1589-0339 Centro Universitário Maurício de Nassau, Brasil

E-mail: samyralima72@gmail.com

Suzy Romere Silva de Alencar

ORCID: https://orcid.org/0000-0002-9547-6727 Universidade Estadual do Piauí, Brasil

E-mail: romeresuzy@gmail.com

Milena Matos da Cruz

ORCID: https://orcid.org/0000-0002-9875-8906 Itpac Porto, Brasi

E-mail: milenamc_5@hotmail.com

João Vitor Messias Esperandio

ORCID: https://orcid.org/0000-0003-0184-4531 Instituto Tocantinense Presidente Antonio Carlos, Brasil

E-mail: jvmessiasesperandio@hotmail.com

Tálison Vieira da Silva

ORCID: https://orcid.org/0000-0002-5628-8694

Faculdade Ademar Rosado, Brasil

E-mail: profesptalison@gmail.com

Maria Clara Cronemberger Guimarães Serzedo

ORCID: https://orcid.org/0000-0002-6237-4097

Universidade Federal do Piauí, Brasil

E-mail: mariaclaraserzedo@gmail.com 


\author{
Regina Célia da Silva \\ ORCID: https://orcid.org/0000-0002-6636-5999 \\ Universidade Federal do Piauí, Brasil \\ E-mail: regina.dasilva@ hotmail.com \\ Brenda Steffane Viana Vasconcelos \\ ORCID: https://orcid.org/0000-0002-9959-2223 \\ Universidade Estadual do Maranhão, Brasil \\ E-mail: bsteffanyv@gmail.com \\ Fares José Lima Morais \\ ORCID: https://orcid.org/0000-0002-9015-1288 \\ Universidade Federal do Delta do Parnaíba, Brasil \\ E-mail: faresjose@uol.com.br \\ José Guilherme Férrer Pompeu \\ ORCID: https://orcid.org/0000-0001-9038-0833 \\ Universidade Federal do Piaú, Brasil \\ E-mail: gpompeu8@gmail.com
}

\begin{abstract}
Resumo
O presente estudo tem como objetivo descrever a doença Sarcoma de Kaposi como uma condição relacionada a pacientes com HIV/AIDS e suas características, além disso o estudo buscou relatar as principais manifestações clinicas da doença, buscando assim, um diagnóstico rápido e efetivo, visando o tratamento para pacientes acometidos pela doença. A metodologia escolhida foi revisão bibliográfica do tipo revisão integrativa da literatura. Para a realização desse estudo, foram consultadas as bases de dados: MEDLINE, Scholar Google, LILACS, SCIELO e Repositórios. Utilizou-se os Descritores em Ciências da Saúde (DeCS) de forma associada: Sarcoma de Kaposi, Vírus da imunodeficiência humana, AIDS, Sintomas e Tratamento. Inicialmente, 205 estudos com a temática proposta; dentre estes, foram excluídos 105 estudos, de acordo com os critérios de exclusão estabelecidos. Após a aplicação dos critérios de inclusão, restaram 12 estudos. O Sarcoma de Kaposi é uma neoplasia pouco prevalente e que há poucos anos era dificilmente vista pela comunidade médica. No entanto, com o aumento da infecção pelo HIV em populações com risco maior de desenvolverem está neoplasia e com os inúmeros diagnósticos tardios da infecção pelo HIV, vem ganhando espaço no sistema de saúde.
\end{abstract}

Palavras-chave: Sarcoma de Kaposi; Vírus da imunodeficiência humana; AIDS; Sintomas; Tratamento.

\begin{abstract}
The present study aims to describe Kaposi's Sarcoma disease as a condition related to patients with HIV/AIDS and its characteristics. Furthermore, the study sought to report the main clinical manifestations of the disease, thus seeking a quick and effective diagnosis, aiming at treatment for patients affected by the disease. The chosen methodology was a bibliographic review of the integrative literature review type. To carry out this study, the following databases were consulted: MEDLINE, Scholar Google, LILACS, SCIELO and Repositories. The Descriptors in Health Sciences (DeCS) were used in an associated way: Kaposi's Sarcoma, Human Immunodeficiency Virus, AIDS, Symptoms and Treatment. Initially, 205 studies with the proposed theme; among these, 105 studies were excluded, according to the established exclusion criteria. After applying the inclusion criteria, 12 studies remained. Kaposi's Sarcoma is a rare neoplasm that a few years ago was hardly seen by the medical community. However, with the increase in HIV infection in populations at higher risk of developing this neoplasm and with the numerous late diagnoses of HIV infection, it has been gaining ground in the health system.
\end{abstract}

Keywords: Kaposi's Sarcoma; Human Immunodeficiency Virus; AIDS; Symptoms; Treatment.

\title{
Resumen
}

El presente estudio tiene como objetivo describir la enfermedad del Sarcoma de Kaposi como una condición relacionada con los pacientes con VIH / SIDA y sus características, además, el estudio buscó reportar las principales manifestaciones clínicas de la enfermedad, buscando así un diagnóstico rápido y efectivo, con el objetivo de tratar la enfermedad. pacientes afectados por la enfermedad. La metodología elegida fue una revisión bibliográfica del tipo revisión integradora de la literatura. Para la realización de este estudio se consultaron las siguientes bases de datos: MEDLINE, Scholar Google, LILACS, SCIELO y Repositories. Los Descriptores en Ciencias de la Salud (DeCS) se utilizaron de forma asociada: Sarcoma de Kaposi, Virus de Inmunodeficiencia Humana, SIDA, Síntomas y Tratamiento. Inicialmente 205 estudios con el tema propuesto; entre estos, se excluyeron 105 estudios, de acuerdo con los criterios de exclusión establecidos. Después de aplicar los criterios de inclusión, quedaron 12 estudios. El sarcoma de Kaposi es una neoplasia poco frecuente que hace unos años apenas era vista por la comunidad médica. Sin embargo, con el aumento de la infección por VIH en poblaciones con mayor riesgo de desarrollar esta neoplasia y con los numerosos diagnósticos tardíos de infección por VIH, ha ido ganando terreno en el sistema de salud.

Palabras clave: Sarcoma de Kaposi; Virus de Inmunodeficiencia Humana; SIDA; Síntomas; Tratamiento. 


\section{Introdução}

O Sarcomade Kaposi (SK) é uma patologia multifocal, conhecida como um tipo raro de neoplasia dos vasos linfáticos e sanguíneos, onde fundamenta-se em nódulos que frequentemente envolvem a pele, mucosas e vísceras. Essa doença foi relatada pela primeira vez em 1872 por Kaposi, um dermatologista da Hungria, como um sarcoma progressivo. Ao contrários do que já se viu em outras neoplasias, o SK pode não resultar de um crescimento de células tumorais de forma autonômica, mas sim em resposta a presença do Herpes vírus tipo 8 (HHV-8) ou KSHV (Assis et al., 2021).

O SK possui uma variação clínica e evolutiva, onde muitas vezes pode apresentar um aspecto de mácula de coloração vermelho-azulada, progredindo para uma placa até chegar em uma forma nodular tumoral de envolvimento profuso, por muitas vezes atua sobre os tecidos cutâneos a vários órgãos particularmente o trato gastrointestinal, linfonodos, pulmões, ossos e mucosa oral (Sousa et al., 2021).

A partir de 1980, com o surgimento da aids, foi descrita uma forma mais agressiva do SK, com uma maior prevalência em homossexuais ou bissexuais masculinos, onde muitos casos estavam relacionados ao vírus de imunodeficiência humana (HIV), logo reconhecida como forma epidêmica do SK, de grande impacto na Saúde Pública por sua alta magnitude e mortalidade. O SK foi a primeira doença oportunista reconhecida em associação com o HIV e ainda é a neoplasia mais frequente relacionada à aids (Tancredi et al., 2017).

Em alguns casos as manifestações clínicas do SK inicia antes mesmo do diagnóstico de AIDS, isso ocorre com maior frequência quando acomete a região cutânea. Tal característica ocorre devido a lesões em placas violáceas e confluentes, as quais podem evoluir para cor acastanhada em diversas partes do corpo. Além disso, pode ainda, agir sobre o trato gastrointestinal (GI), pois nessa região e facilmente identificar casos de mais acometimento visceral, além de baço, fígado ou pulmões. Torna-se mais difícil de diagnosticar a doença quando tal patologia apresenta-se de forma assintomática ou com manifestação gastrointestinal isolada torna-se (Assis et al., 2021).

O SK é uma doença agressiva que pode implicar em complicações, como infecções oportunistas associadas a AIDS, em muitos casos tornando-se fatal. Porém, observa-se que apresenta boa resposta às várias práticas terapêuticas: como excisão cirúrgica, crioterapia e radioterapia, quando as lesões são localizadas e o tratamento sistêmico, reservado para o caso de lesões cutâneas de maior extensão, múltiplas ou com envolvimento de outros órgãos (Borges; Real; Schreiner, 2019).

A partir do que foi apresentado, o presente estudo tem como objetivo descrever a doença Sarcoma de Kaposi como uma condição relacionada a pacientes com HIV/AIDS e suas características, além disso o estudo buscou relatar as principais manifestações clinicas da doença, buscando assim, um diagnóstico rápido e efetivo, visando o tratamento para pacientes acometidos pela doença.

\section{Metodologia}

A metodologia escolhida foi revisão bibliográfica do tipo revisão integrativa da literatura (RIL), que apresenta abordagem qualitativa e abrange os estudos com caráter empírico e teórico, identificando ideias já conhecidas sobre um assunto especifico em estudos realizados e gerando conhecimentos sobre resultados de distintos estudos (Mendes; Silveira; Galvão, 2008).

Para estruturar essa revisão seguiram-se 6 etapas metodológicas: identificação do tema e seleção da hipótese ou questão de pesquisa; estabelecimento de critérios para inclusão e exclusão de estudos; busca na literatura; avaliação dos estudos incluídos na revisão integrativa; Interpretação dos resultados; apresentação da revisão ou síntese do conhecimento. RIL se constitui por seis etapas representadas no Fluxograma 1 abaixo: 
Figura 1: Fluxograma que apresenta o processo de seleção das publicações.

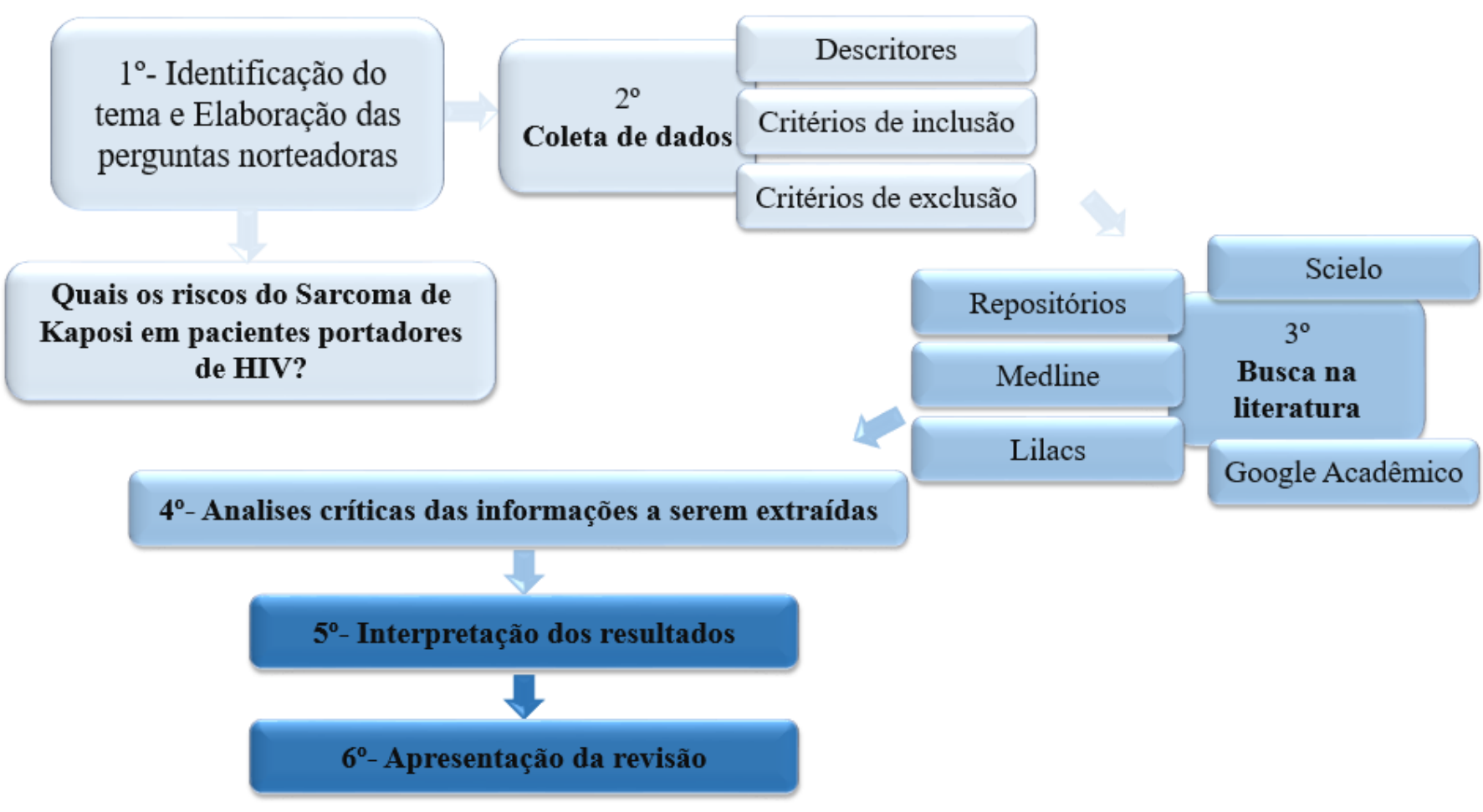

Fonte: Autores (2021).

Para a realização desse estudo, foram consultadas as bases de dados: Medical Literature Analysis and Retrieval System Online (MEDLINE), Scholar Google, Literatura Latino-americana e do Caribe em Ciências da Saúde (LILACS), Scientific Eletronic Library Online (SCIELO) e Repositórios. Utilizou-se os Descritores em Ciências da Saúde (DeCS) de forma associada: Sarcoma de Kaposi, Vírus da imunodeficiência humana, AIDS, Sintomas e Tratamento.

As bases de dados utilizadas foram escolhidas por ser fonte de pesquisa para estudantes e profissionais da área da saúde, por isso, buscou-se identificar as publicações científicas que abordavam o tema deste estudo. Ao final da coleta de dados, foram selecionados os artigos que se adequaram aos objetivos, compondo o corpus de análise.

Os critérios de inclusão foram: artigos disponibilizados na íntegra, disponíveis nos idiomas português e inglês e publicados entre os anos de 2015 e 2021. Os critérios de exclusão foram: artigos incompletos, cartas ao editor, debates, resenhas, resumos ou artigos publicados em anais de eventos, indisponíveis na íntegra e duplicados.

Para a análise crítica das literaturas foi realizada a análise de Bardin (2011), que consiste em um método que abrange todas as classes de documentos e textos e dividido em três etapas:

1. Pré-análise, nessa etapa ocorreu o acesso as literaturas por meio dos bancos de dados utilizando os descritores e filtros referentes aos critérios de inclusão e exclusão, com intuito de organizar o material a ser utilizado tornando-o operacional;

2. Exploração do material, ocorre a etapa de codificação do material selecionado, nessa fase foi realizado o recorte das unidades de registro, e a categorização em eixos temáticos;

3. No tratamento dos resultados, consistiu na interpretação dos dados em que foram reunidas por características comuns facilitando a apresentação dos resultados através da interpretação de interferência, cuja interpretação consistiu na apresentação dos estudos selecionados e explanação dos eixos temáticos.

A análise e a interpretação dos dados foram feitas por meio da descrição dos achados. Os dados foram organizados em uma tabela Excel®, que compreendeu as seguintes colunas de sintetização: título do estudo, base de dados, periódico, ano de 
publicação, contexto/local de estudo, desenho metodológico, resultados e conclusões.

Cabe salientar que os aspectos éticos e os direitos de autoria foram devidamente respeitados, por meio da referenciação dos autores dos trabalhos utilizados. Por se tratar de um estudo de revisão de literatura, não houve necessidade de apreciação por um Comitê de Ética em Pesquisa (CEP).

\section{Resultados e Discussão}

Foram considerados, inicialmente, 205 estudos com a temática proposta; dentre estes, foram excluídos 105 estudos, de acordo com os critérios de exclusão estabelecidos. Após a aplicação dos critérios de inclusão, restaram 12 estudos. Com os estudos incluídos foram feitas revisões críticas e corroborativas sobre cada um deles.

\section{Tabela 1.}

TITULO

SARCOMA DE KAPOSI:

CARACTERÍSTICAS CLÍNICOLABORATORIAIS,

ESTADIAMENTO INICIAL E DESFECHO EM PACIENTES COM AIDS ATENDIDOS EM CENTRO DE REFERÊNCIA DE RECIFE/PE NO PERÍODO DE 2004 A 2014

SARCOMA DE KAPOSI EM PACIENTES HIV: NOVAMENTE UMA REALIDADE.

PERFIL EPIDEMIOLÓGICO DO SARCOMA DE KAPOSI EM PACIENTES COM HIV/AIDS POPULAC̄̃̃O BRASILEIRA REVISÃO DE LITERATURA

HIV-ASSOCIATED KAPOSI

SARCOMA AND RELATED DISEASES.

ESTUDO CLÍNICO DE SARCOMA DE KAPOSI EM PACIENTES COM HIV/AIDS, DE 1985-1994 E 2005-2014.

SARCOMA DE KAPOSI EPIDÊMICO ASSOCIADO AO VÍRUS HERPES HUMANO TIPO-8, UMA REVISÃO.

RECENT ADVANCES IN HIVASSOCIATED KAPOSI SARCOMA.

PREVALÊNCIA DE SARCOMA

DE KAPOSI EM PACIENTES COM AIDS E FATORES

AUTORES / ANO OBJETIVO

\section{CONCLUSÃO}

Descrever as principais características clínicolaboratoriais, o estadiamento

(Lima, 2015) inicial e desfecho do sarcoma de Kaposi em pacientes com aids atendidos no HC-UFPE no período de 2004 a 2014.

Revisar na literatura o Sarcoma de Kaposi em pacientes com (Borges; Real; Schreiner, HIV/SIDA, descrevê-lo, apontar 2019).

sua clínica a fim de auxiliar no diagnóstico e apresentar as opções de tratamento.

Estabelecer o perfil

(Pereira e Torres, 2015) epidemiológico do SK em pacientes com HIV/AIDS na população brasileiras

\section{(Gonçalves; Uldrick;} Yarchoan, 2017).

(Maldonado et al., 2014).

(Castro et al., 2016)

(Pria et al., 2019).

(Tancredi et al., 2017).
Descrever sobre o Sarcoma de

Kaposi associado ao HIV e doenças relacionadas

O objetivo do estudo em questão foi estabelecer a prevalência de

SK em pacientes com AIDS atendidos na Disciplina de Doenças Infecciosas e

Parasitárias do Hospital Universitário Pedro Ernesto, da Universidade do Estado do Rio de Janeiro em dois cortes temporais distintos (1985-1994 e 2005-2014).

Realizar uma revisão bibliográfica sobre os aspectos mais relevantes do Sarcoma de Kaposi associado ao vírus do herpes humano tipo 8

Descrever os avanços recentes no sarcoma de Kaposi associado ao HIV

Estimar a prevalência de

sarcoma de Kaposi (SK) em pacientes com aids e identificar
Entre os casos estudados, oito tiveram desfecho letal $(36,5 \%)$. A despeito do diagnóstico tardio do SK associado à aids na população estudada, inclusive com risco desfavorável no momento do estadiamento, encontrou-se mortalidade menor do que a reportada em outros estudos e que pode estar relacionado ao acesso a serviço de saúde especializado.

O Sarcoma de Kaposi é uma neoplasia pouco prevalente, porém, com o aumento da infecção pelo HIV

diagnosticada tardiamente, vem ganhando espaço no sistema de saúde, sendo necessário saber o identificar, uma vez que o diagnóstico e o tratamento precoce podem diminuir a morbimortalidade das pessoas com HIV.

O perfil epidemiológico do SK epidêmico no Brasil é formado em sua maioria por homens, adultos jovens, brancos, tendo como principais fatores de risco a homossexualidade e bissexualidade, bem como uso de drogas injetáveis e não uso da HAART. Há necessidade de realizar estudos que abordam a população brasileira como um todo a fim de buscar maior gama de informações que viabilizem uma melhor discussão acerca dos pacientes com SK associado a AIDS no Brasil.

A descoberta do KSHV e sua identificação como causa do KS abriu a porta para uma série de linhas de investigação e novos insights. Reconhece-se agora que o KSHV pode causar várias doenças, várias das quais não tinham sido reconhecidas anteriormente.

O SK na forma disseminada foi predominante em ambos os grupos. Houve uma diminuição significativa na prevalência de casos de SK desde o início da epidemia do HIV/AIDS até os dias atuais, provavelmente pelo uso dos antirretrovirais. Houve uma maior frequência da população masculina e de casos de SK na forma disseminada

A partir da presente pesquisa bibliográfica pode se inferir que esta neoplasia aqui retratada ganhou importância a partir da epidemia HIV/SIDA na década de 80, desde sua possível associação com a infecção pelo HIV. Apesar de ocorrer entre homens e mulheres, ainda há uma maior prevalência nos homens.

A introdução do cART não resolveu o desafio do SK associado ao HIV.

o SK tem alta prevalência em São Paulo-SP; estratégias para o diagnóstico precoce do HIV podem resultar em diminuição desta prevalência. 


\begin{tabular}{|c|c|c|}
\hline ASSOCIADOS & & $\begin{array}{l}\text { os fatores associados à } \\
\text { ocorrência da neoplasia. }\end{array}$ \\
\hline $\begin{array}{c}\text { PREVALÊNCIA DE SARCOMA } \\
\text { DE KAPOSI EM ASSOCIAÇÃO A } \\
\text { IMUNODEFICIÊNCIA } \\
\text { ADQUIRIDA EM PACIENTES } \\
\text { ATENDIDOS NO HOSPITAL } \\
\text { REGIONAL DE CÁCERES-MT }\end{array}$ & (Marques; Garcia, 2016). & $\begin{array}{l}\text {.O objetivo deste trabalho é o de } \\
\text { ressaltar a importância do SK no } \\
\text { contexto da pandemia de AIDS }\end{array}$ \\
\hline $\begin{array}{c}\text { ABORDAGEM TERAPÊUTICA } \\
\text { DO SARCOMA DE KAPOSI-A } \\
\text { IMPORTÂNCIA DA } \\
\text { MULTIDISCIPLINARIDADE. }\end{array}$ & (Mansinho et al., 2015). & $\begin{array}{c}\text { Realizar Uma Abordagem } \\
\text { Terapêutica Do Sarcoma De } \\
\text { Kaposi-A Importância Da } \\
\text { Multidisciplinaridade. }\end{array}$ \\
\hline $\begin{array}{c}\text { SARCOMA DE KAPOSI EM } \\
\text { PACIENTES HIV: NOVAMENTE } \\
\text { UMA REALIDADE. }\end{array}$ & (Borges et al., 2014). & $\begin{array}{l}\text { Revisar na literatura o Sarcoma } \\
\text { de Kaposi em pacientes com } \\
\text { HIV/SIDA, descrevê-lo, apontar } \\
\text { sua clínica a fim de auxiliar no } \\
\text { diagnóstico e apresentar as } \\
\text { opções de tratamento. }\end{array}$ \\
\hline $\begin{array}{c}\text { CLINICAL AND LABORATORY } \\
\text { PROFILE OF PEOPLE LIVING } \\
\text { WITH HIV/AIDS WITH ORAL } \\
\text { KAPOSI SARCOMA. }\end{array}$ & (Alves et al., 2021). & $\begin{array}{c}\text { Avaliar o perfil clínico e } \\
\text { laboratorial de pessoas com } \\
\text { sarcoma de Kaposi oral (SK) } \\
\text { associado à AIDS (KS-AIDS), } \\
\text { acompanhadas em um hospital } \\
\text { público universitário de } \\
\text { Salvador, Bahia, Brasil, nos } \\
\text { últimos } 10 \text { anos. }\end{array}$ \\
\hline
\end{tabular}

Fonte: Autores (2021).
Por fim, de maneira geral e até o momento, o que se deve buscar é o controle da doença e a paliação eficaz dos sintomas. Como prevenção da doença AIDS está relacionado como única opção o uso de preservativos, tanto masculino, quanto feminino, disposto em postos de saúde, hospitais e centro de tratamento

Destaca-se a importância da abordagem multidisciplinar

da doença, bem como o actual estado da arte nas terapêuticas existentes, sistematizando e estratificando as opções disponíveis na prática clínica.

O Sarcoma de Kaposi é uma neoplasia pouco prevalente, porém, com o aumento da infecção pelo HIV diagnosticada tardiamente, vem ganhando espaço no sistema de saúde, sendo necessário saber o identificar, uma vez que o diagnóstico e o tratamento precoce podem diminuir a morbimortalidade das pessoas com HIV.

O SK oral associado à AIDS foi frequente em homens jovens adultos negros, em sua maioria com lesões manifestadas no mesmo período do diagnóstico da infecção pelo HIV, já em estágio tardio, e associado a imunossupressão grave e alta carga viral.

Por mais de meio século o SK foi considerado doença rara, que acometia principalmente idosos do sexo masculino. Por meio da análise de dados epidemiológicos de cinco diferentes grupos populacionais, apareceram outras vias para a melhor compreensão desta neoplasia. Nos últimos 30 anos, outras apresentações de SK foram descritas com a descrição de três novos tipos além do SK clássico: SK endêmico africano, SK iatrogênico (após transplante) e o SK epidêmico, associado à aids (Lima, 2015).

Em 1872, Morris Kaposi descreveu uma patologia definida por múltiplas lesões hiperpigmentadas, nodulares, onde acomete principalmente as extremidades dos membros inferiores. Hoje, a forma epidêmica da doença está diretamente associada com pacientes acometidos pelo vírus HIV, além disso essa forma é conhecida por ser a mais agressiva e a principal doença neoplásica associada a esses pacientes (Borges et al., 2019).

Pereira e Torres (2015), ressaltaram em sua pesquisa sobre a descoberta da presença de DNA de um novo tipo de herpes vírus em amostras cutâneas de Sarcoma de Kaposi. A partir disso, todas as formas do SK estão diretamente relacionadas a existência do Herpes Vírus Humano 8 (HHV-8), confirmando assim, ser uma estado necessária para o progressão da neoplasia, porém não é fator suficiente. O HHV-8 é um vírus sexualmente transmissível, onde aproximadamente 95\% dos pacientes apresentam o HHV-8 nas lesões de SK.

Microscopicamente, as lesões ocasionadas pelo SK em células tumorais fusiformes, em sua maior parte está acompanhadas por fibrose, infiltrados inflamatórios, fendas vasculares e hemossiderina. A coloração imunohistoquímica para CD31 é positiva, e a coloração de células fusiformes para herpesvírus associado ao SK é sensível e específica (figura 1). Os glóbulos vermelhos extravasados dão às lesões uma tonalidade púrpura. Há relatos de que no início do curso do SK, os tumores são multiclonais. Porém, já foi descrito que a doença quando está em estado mais avançado é oligoclonal ou monoclonal, e a clonalidade do SK ainda é uma área de incerteza (Gonçalves et al., 2017). 
Figura 1. Sarcoma de Kaposi cutâneo, aumento de 200x.

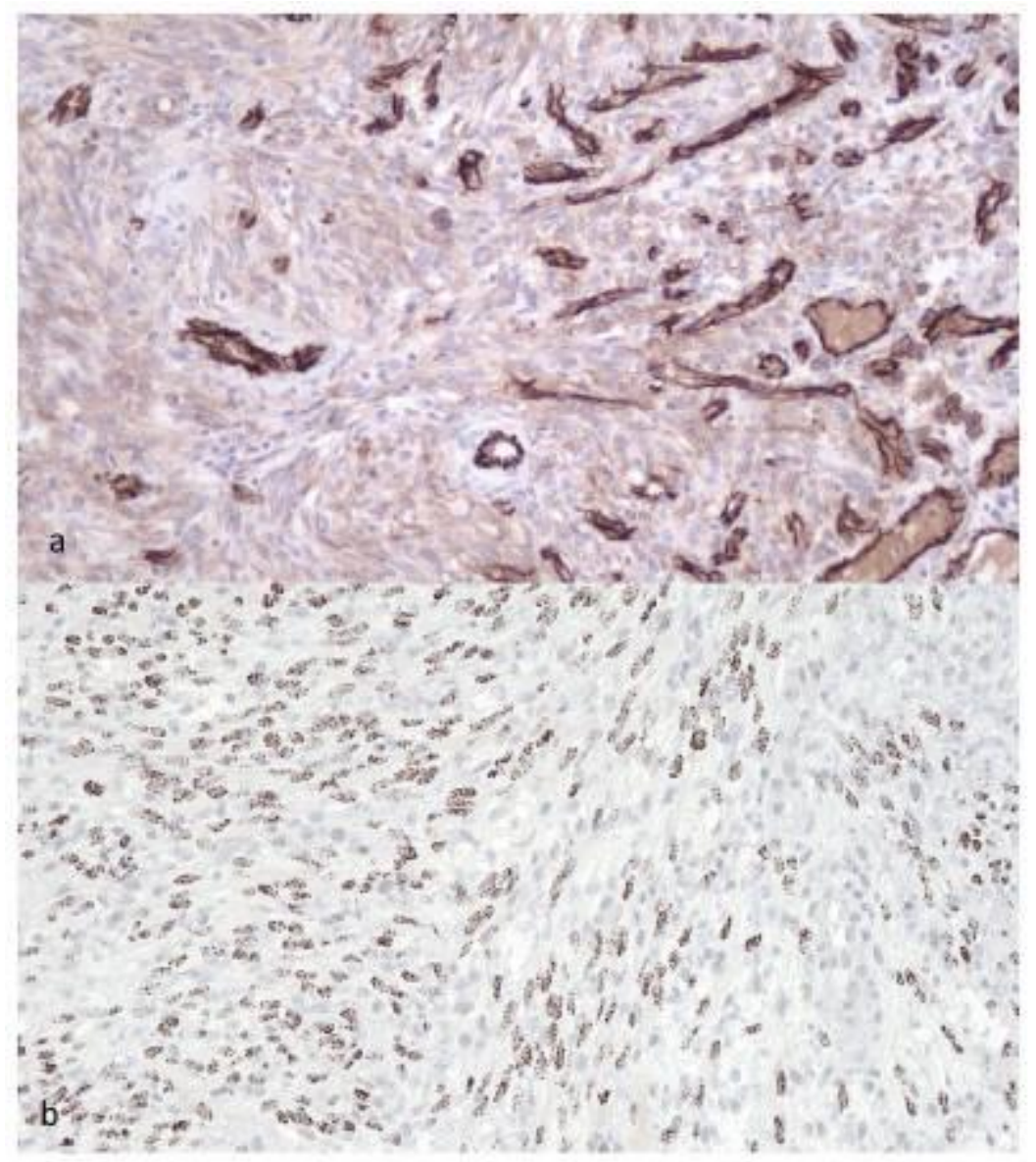

a) tumor de células fusiformes com vasos sanguíneos anormais destacados por imuno-histoquímica de CD31, b) células fusiformes KS com coloração nuclear de antígeno nuclear codificado por latência codificado por KSHV. Fonte: Adaptado de (Gonçalves et al., 2017).

A situação imunológica do hospedeiro e o acometimento dos linfonodos são fatores essenciais no prognóstico do paciente. O SK pode ser vista em até seis formas de formas clínicas, que são: a forma macular, a forma em placa, a nodular, a exofítica, a infiltrativa e a linfadenopática. As lesões do SK são altamente angiogênicas e, como resultado, são geralmente vermelhas, roxas, ou na cor marrom. Além disso, podem ser dolorosas, principalmente se causarem edema na pele próxima não afetada. A progressão das lesões do SK é ocorre principalmente na pele, que é o órgão mais afetado, seguido por manifestações pulmonares e digestivas. A partir disso, sabe-se que a mortalidade do SK está diretamente relacionada a infecções oportunistas, a hemorragias gastrointestinais e a complicações pulmonares (Maldonado et al., 2014).

Ao se comparar as lesões da forma clássica com aquelas epidêmicas relacionadas ao HIV, observa-se que neste último grupo as lesões apresentam principalmente a forma disseminada, porém em relação ao tamanho apresentam em menor proporção, menos violáceas, pouco infiltradas e, em alguns casos, nodulares. Em mais de $60 \%$ é comum a presença de linfadenopatia generalizada no momento do diagnóstico, e cerca de 50\% dos pacientes têm lesões em um ou mais sítios ao longo do tubo digestório (Maldonado et al., 2014).

Mesmo com os progressos de novas tecnologias voltada ao manejo sistêmico do SK, até $15 \%$ dos pacientes desenvolvem doença progressiva, apesar de alcançar um bom controle imunovirológico do HIV com RNA HIV-1 plasmático indetectável e contagens adequadas de células T CD4 (> $\left.300 / \mathrm{mm}^{3}\right)$. A imunopatologia do SK refratário a TARc é de alta complexibilidade. Estudos mecanísticos descrevem que este tipo de doença está associado à senescência imune a células $\mathrm{T}$ como um traço fisiopatológico chave, onde muito desses pacientes tem um sentido clínico indolente semelhante ao do subtipo clássico. A alternância sequencial de agentes quimioterápicos sem reação cruzada foi vista com bons olhos, pois tornou-se 
eficaz no controle do HIV-SK em uma série de 20 pacientes (Pria et al., 2019).

Segundo Lima (2015), no ponto de vista clínico, o grau de acometimento do SK é possui variações, porém na maior parte dos casos cursa com alta morbidade e mortalidade. Diversos estudos tem buscado descrever as características clínicas da doença. Porém, ainda e escasso a quantidade de artigos que tratem sobre o estadiamento na ocasião do diagnóstico e desfecho destes pacientes. O mesmo estudo citou uma pesquisa onde foi observando apenas a forma clínica visceral entre 319 pacientes com SK que apresentaram células T-CD4+ com discreta variação entre 197 e 157 céls/mm3, onde concluíram que não houve diferença no desfecho desta forma clínica, quando comparada com o grupo que apresentava exclusivamente lesões cutâneas.

No estudo realizado por Maldonado et al. (2014), onde foi descrito um corte temporal entre os anos de 1985 a 1994, onde buscou relatar os primeiros anos de atendimento de pacientes com AIDS na Disciplina de Doenças Infecciosas e Parasitárias do HUPE-UERJ, e um segundo corte temporal entre 2005 a 2014. A partir disso, foram selecionados 81 pacientes com AIDS, sendo estes adultos de ambos os sexos, com diagnóstico de SK. Quanto à forma de apresentação clínica do SK de acordo com a extensão da lesão (Figura 1), a forma disse minada predominou em ambos os grupos, com valores percentuais de 73\% (1985-1994) e 72\% (2005-2014).

Figura 2. Formas clínicas de apresentação das lesões do sarcoma de Kaposi.
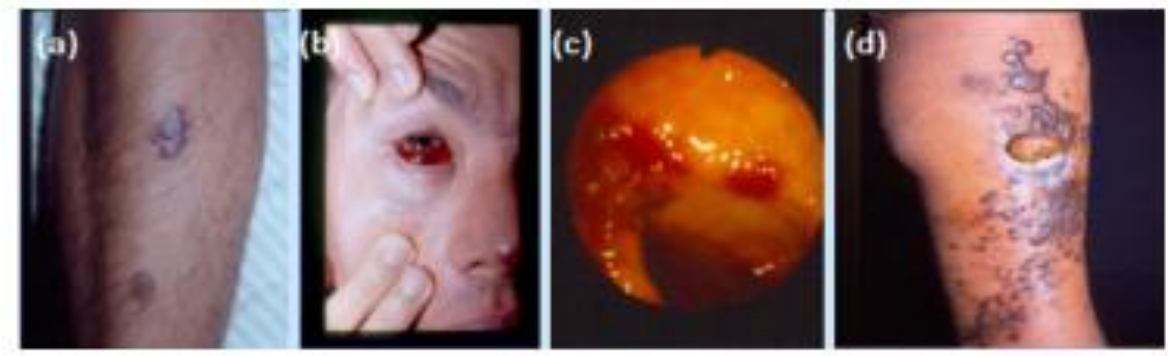

(a) lesão na pele - localizada, (b) lesão na pele e conjuntva - disseminada, (c) lesão no cólon - disseminada, e (d) lesão na pele disseminada. Fonte: Adaptado de (Maldonado et al., 2014).

Observou-se que o SK associado à AIDS está presente principalmente em homens que fazem sexo com homens (HSH), sugerindo uma segunda etiologia infecciosa. Em 1994, usando a análise de diferença representacional, foi identificado um novo gama-herpesvírus em um tumor KS associado à AIDS. Este vírus, mais intimamente relacionado ao vírus EpsteinBarr (EBV), foi denominado herpesvírus associado ao sarcoma de Kaposi (KSHV). Outras pesquisas descrevem que o KSHV, também conhecido herpesvírus humano-8 (HHV-8), é o agente etiológico de todos os subtipos epidemiológicos de KS (Gonçalves et al., 2017).

As lesões ocasionadas pelo SK são mediadas através de citocinas inflamatórias e proteínas que promovem as proliferações celulares, e ambas são desenvolvidas pelo KSHV, que quando agregado ao HIV, apresentam-se de um modo mais agressivo e surge como um dos primeiros sintomas da AIDS (Figura 3) (Castro et al., 2016). 
Figura 3. Proliferação/ de células endoteliais a partir da infecção pelo KSHV.

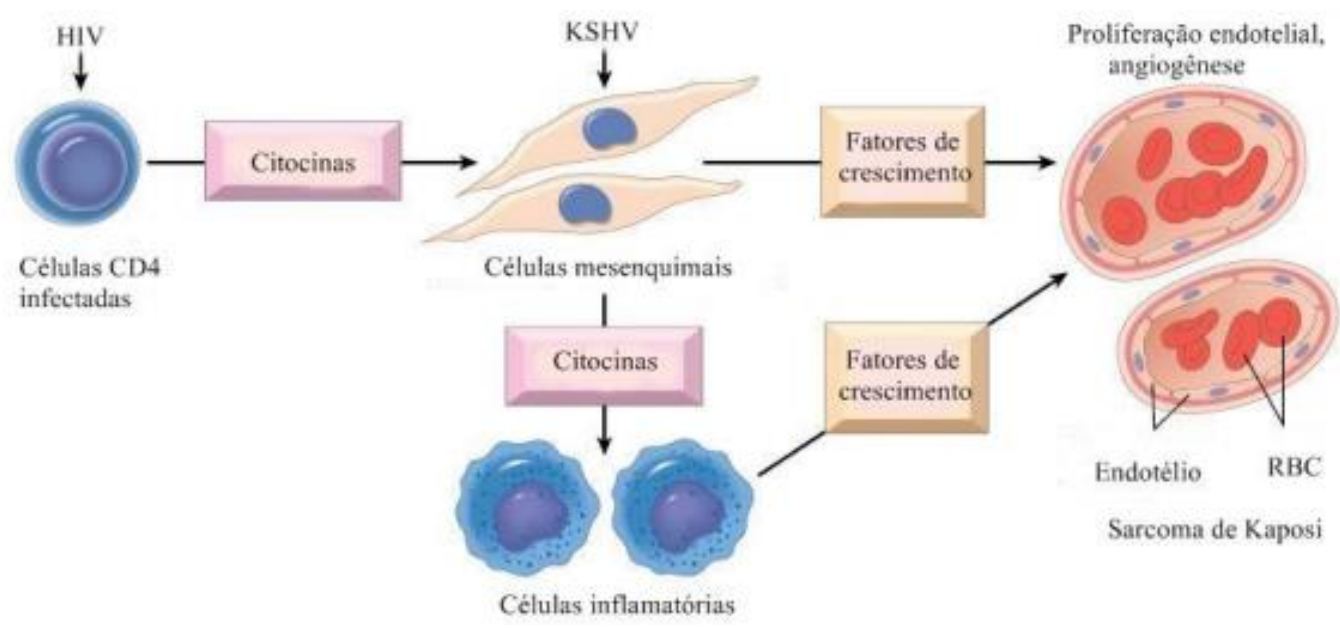

Fonte: Adaptado de (Castro et al., 2016).

Quando paciente e acometido pelo HIV não tratado, o diagnóstico de SK oral é essencial como um marcador prognóstico de sobrevida. Sem diagnóstico e, consequentemente, sem tratamento adequado, os pacientes podem progredir para quadro de complicações relacionadas à imunossupressão desencadeada pelo HIV. A partir disso, a identificação das lesões bucais do KS-AIDS pelo profissional é de grande valia para o diagnóstico precoce, ou para a detecção de imunossupressão e progressão da AIDS (Alves et al., 2021).

O tratamento da AIDS associada ao SK, bem como o inverso deve ser realizado na supressão da replicação do HIV iniciando ou dando continuidade à terapia antirretroviral. As taxas de SK regrediram de maneira significativa desde o advento da Terapia Antirretroviral Altamente Ativa (HAART). A escolha de um tratamento deve ser realizado com cuidado rigoroso e individualizado, onde deve-se considerar alguns fatores como gravidade do caso e localização das lesões (Pereira; Torres, 2015).

Com a introdução da HAART, foi possível identificar melhora do sistema imune e diminuição no risco de desenvolver SK entre pessoas acometidas pelo HIV. Porém, o SK ainda é visto como o tumor de maior relevância nessa população. Apesar de a HAART não atuar sobre a replicação do HHV-8, a diminuição da lesão de SK-aids têm sido observada rotineiramente após o uso da associação de HAART com quimioterapia ou radioterapia, permanecendo controverso o mecanismo pelo qual a HAART leva à regressão do SK (Tancredi et al., 2017).

Inicialmente teve-se medo da implementação da terapêutica com drogas citotóxicas, devido ao paciente com SK/SIDA ser um indivíduo com uma imunodeficiência de base. Por isso, buscou-se o uso de drogas combinadas, e, nas doses usuais, dá-se preferência à monoterapia ou à associação de fármacos com doses diminuídas. Dentre os agentes quimioterápicos de uso sistêmico, os mais extensivamentes estudados têm sido os alcalóides da vinca (Vincristina e Vimblastina), a bleomicina e as antraciclinas (Daunorubicina e Doxorubicina) (Marques \& Garcia, 2016).

Quando se fala sobre à intervenção terapêutica, a dermatologia tem um papel importante nas formas cutâneas, com uma abordagem essencialmente local, sendo realizada, a partir de um seguimento regular para monitorizar a resposta, programar as intervenções e detectar as recidivas. Quando existe um agravamento sistémica ou local, apesar da terapêutica escolhida, os doentes devem ser abordados a partir de reuniões multidisciplinares, onde deve-se ser realizada uma decisão partilhada relativamente ao tratamento, que pode passar pela Radioterapia ou terapêutica citotóxica na Oncologia, devendo ser avaliada resposta regularmente quer clínica quer imagiologicamente, particularmente na doença visceral (Mansinho et al., 2015). 
Não há uma prevenção específica descoberta até hoje voltada ao SK. Um agente anti-herpes vírus, o ganciclovir, faz com que a taxa de incidência de SK caia de maneira significativa, isso é visto também em indivíduos com infecção pelo HIV tratados por retinite e citomegalovírus. Porém deve-se ter cuidado, pois o uso de maneira incorreta desses medicamento em pacientes com HIV ou outras populações com infecção pelo KSHV não é recomendado devido à sua toxicidade (Borges et al., 2014).

\section{Considerações Finais}

O Sarcoma de Kaposi é uma neoplasia pouco prevalente e que há poucos anos era dificilmente vista pela comunidade médica. No entanto, com o aumento da infecção pelo HIV em populações com risco maior de desenvolverem está neoplasia e com os inúmeros diagnósticos tardios da infecção pelo HIV, vem ganhando espaço no sistema de saúde.

Em relação a prevalência de casos de sarcoma de Kaposi em pacientes com HIV/AIDS observa-se que há uma maior frequência da população masculina e de casos de sarcoma de Kaposi disseminado. Entretanto, é importante enfatizar que, mesmo tendo diminuído os casos de SK, está ainda é uma forma de neoplasia frequente em pacientes com HIV/AIDS e o diagnóstico tem de ser pensado independentemente do sexo.

Contudo, há pacientes HIV-positivos que mesmo após o tratamento HAART, o SK ainda persiste. Acredita-se que este fenômeno pode aumentar à medida que a população envelhece infectada com HIV. Dessa forma, é importante acompanhar o SK, visto que essa doença continua sendo um problema da saúde, bem como para evitar o aumento da sua incidência

O diagnóstico preciso e precoce desta neoplasia por meio da associação clínica e histopatológica tem finalidade terapêutica imediata e possui importância fundamental na diminuição da morbimortalidade em pacientes que possuem a forma epidêmica da doença.

\section{Referências}

Alves, C. G. B., Assis, M. S., Maciel, A. D. S., da Silva, J. C., Leite-Ribeiro, P. M., Lins-Kusterer, L., \& Sarmento, V. A. (2021). Clinical and laboratory profile of people living with HIV/AIDS with oral Kaposi sarcoma. AIDS research and human retroviruses.

Assis, Y. D., de Melo, L. L. P., de Rezende, L. J. S., Oliveira, E. F. P., Campos, L. C. O., Rocha, A. G. S., ... \& Rodrigues, A. V. (2021). Sarcoma de Kaposi Gastrointestinal primário: relato de caso. Revista Eletrônica Acervo Saúde, 13(1), e5349-e5349.

Bardin, L. (2011). Análise de Conteúdo. Ed. Revista e Ampliada.

Borges, S. A. L., Real, L. H. G., \& Schreiner, R. B. (2019). Sarcoma de Kaposi em pacientes HIV: novamente uma realidade. Revista Eletrônica Acervo Saúde, 11(6), e352-e352.

Borges, A. O., Pereira, L. G., Humberto, J. V., \& Danziger, L. R. (2021). Sarcoma De Kaposi E O Hiv: Uma Revisão Literária. Revista Saúde Multidisciplinar, 10(2).

Castro, V. M. D. D. (2016). Sarcoma de kaposi epidêmico associado ao vírus herpes humano tipo-8, uma revisão. Trabalho de Conclusão de Curso. Universidade Federal de Campina Grande. CUITÉ - PB. Brasil.

Gonçalves, P. H., Uldrick, T. S., \& Yarchoan, R. (2017). HIV-associated Kaposi sarcoma and related diseases. AIDS (London, England), 31 (14), 1903.

Lima, C. T. D. (2015). Sarcoma de Kaposi: características clínico-laboratoriais, estadiamento inicial e desfecho em pacientes com AIDS atendidos em centro de referência de Recife/PE no período de 2004 a 2014 (Master's thesis, Universidade Federal de Pernambuco).

Maldonado, G., Júnior, O. N., Arnóbio, A., Alfradique, G. R., Ornellas, M. H., \& de Lima, D. B. (2015). Estudo clínico de sarcoma de Kaposi em pacientes com HIV/AIDS, de 1985-1994 e 2005-2014. Revista Hospital Universitário Pedro Ernesto (HUPE), 14.

Mansinho, A., Macedo, D., Nunes, B., Fernandes, I., Jorge, M., \& Borges-Costa, J. (2015). Abordagem Terapêutica Do Sarcoma De Kaposi-A Importância Da Multidisciplinaridade. Journal of the Portuguese Society of Dermatology and Venereology, 73(2), 199-208.

Marques, E. G., \& Garcia, E. S. (2016). Prevalência de sarcoma de Kaposi em associação a imunodeficiência adquirida em pacientes atendidos no Hospital regional de Cáceres-MT. Revista Saberes da FAPAN. v, 3(1), 74-81.

Mendes, K. D. S., Silveira, R. C. D. C. P., \& Galvão, C. M. (2008). Revisão integrativa: método de pesquisa para a incorporação de evidências na saúde e na enfermagem. Texto \& contexto-enfermagem, 17(4), 758-764.

Pereira, K. G., \& Torres, O. D. D. S. (2015). Perfil epidemiológico do Sarcoma de Kaposi em pacientes com HIV/AIDS população brasileira: revisão de 
Research, Society and Development, v. 11, n. 2, e9911225305, 2022

(CC BY 4.0) | ISSN 2525-3409 | DOI: http://dx.doi.org/10.33448/rsd-v11i2.25305

literatura. SEMOC-Semana de Mobilização Científica-Perfil epidemiológico do Sarcoma de Kaposi em pacientes com HIV/AIDS população brasileira: revisão de literatura.

Pria, A., Pinato, D. J., Bracchi, M., \& Bower, M. (2019). Recent advances in HIV-associated Kaposi sarcoma. F100oResearch, 8.

Sousa, R. H. A. D. (2021). Desenvolvimento do sarcoma de Kaposi oral associado a carga viral do HIV, CD4+ e razão da contagem CD4+/CD8+: Oral Kaposi sarcoma development is associated with HIV viral load, CD4+, and CD4+/CD8+ ratio count.

Tancredi, M. V., Pinto, V. M., Silva, M. H. D., Pimentel, S. R., Silva, T. S. B. D., Ito, S. M. A., \& Toscano, A. L. D. C. C. (2017). Prevalência de sarcoma de Kaposi em pacientes com aids e fatores associados, São Paulo-SP, 2003-2010. Epidemiologia e Serviços de Saúde, 26, $379-387$. 